\title{
Critical Temperature for Bose-Einstein condensation in quartic potentials
}

\author{
S. Gautan? and D. Angom t \\ Physical Research Laboratory \\ Navarangpura, Ahmedabad - 380009
}

\begin{abstract}
The quartic confining potential has emerged as a key ingredient to obtain fast rotating vortices in BEC as well as observation of quantum phase transitions in optical lattices. We calculate the critical temperature $T_{c}$ of bosons at which normal to BEC transition occurs for the quartic confining potential. Further more, we evaluate the effect of finite particle number on $T_{c}$ and find that $\Delta T_{c} / T_{c}$ is larger in quartic potential as compared to quadratic potential for number of particles $<10^{5}$. Interestingly, the situation is reversed if the number of particles is $\gtrsim 10^{5}$.
\end{abstract}

PACS numbers: 03.75.Hh,05.30.Jp

\section{INTRODUCTION}

Particles in anharmonic potentials is a well studied example of chaotic system. A simple but good model of a system which exhibits parametric transitions from regular to chaos and vice-versa is the quartic oscillator. The parameters which control the transitions are the coupling strengths of the cross terms. The quartic oscillator besides being a case study of chaotic system, plays a very important role in the guise of Higg's field, through which all the known fundamental particles acquire finite masses. The same potential also appears in optical lattices, where quantum phase transition from superfluid to Mott insulator has been observed [1]. Optical lattices are regular intensity patterns of light created with counter propagating laser beams. The laser beams have a Gaussian profile and create an overlapping anharmonic potential across the optical lattice. Recent theoretical studies show that realization of the quantum phase transition can be more robust with a quartic potential [2]. Besides the quantum phase transitions, introducing a quartic confining potential stabilizes fast rotating vortices in Bose-Einstein condensates [3, 4, ,5, 6, 7, 8]. In a recent work [9], the condensation temperature and thermodynamic properties of a rotating ideal Bose gas in an anharmonic trap has been studied. However, in the experimental realization of the quadratic plus quartic confining potential, the observation of the fast rotating vortices eludes an unambiguous detection [10].

In this paper we calculate the critical temperature $T_{c}$ at which bosons confined by a quartic potential condense. BEC in such a potential was studied for low-dimensional systems [11]. This calculation requires the density of states. In our calculations we use the semiclassical expression of the density of states, which is valid at higher energies. It is however not appropriate to study low lying states. In particular, the energy of the ground state is essential to estimate $T_{c}$ for finite number of particles. To

\footnotetext{
*Electronic address: sandeep@prl.res.in
}

${ }^{\dagger}$ Electronic address: angom@prl.res.in estimate the correction to $T_{c}$ in the finite particle case, we calculate the ground state energy analytically [12]. The calculation is based on a method which optimizes the matrix elements of the quartic potential Hamiltonian in the harmonic oscillator basis.

Our calculations show that $T_{c}$ in quartic confining potential is higher than quadratic potential. This is perhaps to be expected, since $T_{c}$ varies as $N^{1 / \alpha}$ when the density of states is proportional to $\epsilon^{\alpha-1}$. In case of 3D harmonic oscillator potential $\alpha=3$ whereas it is $9 / 4$ in the case of $3 \mathrm{D}$ quartic potential. Hence, in the quartic potential case $T_{c}$ varies as $N^{4 / 9}$ compared to $N^{1 / 3}$ in the case of harmonic potential. From our calculations, it is evident that the cross terms increase $T_{c}$ in $2 \mathrm{D}$ as well as $3 \mathrm{D}$ quartic potential. We find that $T_{c}$ rises by factor of 1.2 and 1.1 in the $3 \mathrm{D}$ and $2 \mathrm{D}$ potentials respectively. However, the experimental realizations of the trapping potentials, which are created from laser beams, are more appropriately described without the cross terms.

\section{II. $T_{c}$ FOR 3D QUARTIC POTENTIAL TRAP}

The general form of the quartic oscillator potential is $\lambda(\boldsymbol{r} . \boldsymbol{r})^{2}$. It is homogeneous and has cross terms in the Cartesian coordinate representation which couple motions along different axes. Potentials of this form occur in optical lattices, where counter propagating lasers create undulating patterns of standing radiation field. In one dimension, a pair of counter propagating Gaussian laser beams along $z$-axis of intensity profile $I_{0} \exp \left(-2 r^{2} / w\right)$ creates an array of periodic intensity minima and maxima. These are located along the $z$-axis. Depending on the detuning of the laser, the atoms are attracted to the intensity minima or maxima. Usually the wavelength of the laser $\lambda$ is much smaller than the beam width $w$. To a very good approximation, the potential across a surface normal to the laser beam

$$
V(r)=I_{0}\left(\frac{-2 r^{2}}{w}+\frac{4 r^{4}}{w^{2}}\right) .
$$

Tuning the parameters of the laser beams, it possible to retain only the quartic term. For simplicity, neglecting 
the cross terms, in three dimension

$$
V(x, y, z)=\lambda\left(x^{4}+y^{4}+z^{4}\right) .
$$

The eigen energies of the corresponding Hamiltonian is the sum of eigen values corresponding to each dimension. For the one dimensional quartic oscillator, eigen values can be calculated by minimizing the expectation of the Hamiltonian in the basis states of harmonic oscillator of appropriately chosen frequency[12]. Generalizing the result to three dimensional case, the eigen energy

$$
\epsilon\left(n_{1}, n_{2}, n_{3}\right)=1.389 \sum_{i=1}^{3}\left(n_{i}+\frac{1}{2}\right)^{\frac{4}{3}}\left(\frac{\lambda \hbar^{4}}{m^{2}}\right)^{\frac{1}{3}} .
$$

Since $1.389\left(\lambda \hbar^{4} / m^{2}\right)^{1 / 3}$ has the dimensions of energy we can represent this factor by $\hbar \omega$, then

$$
\epsilon\left(n_{1}, n_{2}, n_{3}\right)=\sum_{i=1}^{3}\left(n_{i}+\frac{1}{2}\right)^{\frac{4}{3}} \hbar \omega .
$$

We now determine the number of states $G(\epsilon)$ with energy less than a given value $\epsilon$. For energies large compared to $\hbar \omega$, we may treat $n_{i}$ 's as continuous variables and neglect the ground state energy. To calculate $G(\epsilon)$, we introduce a coordinate system in terms of the three variables $\epsilon_{i}=$ $n_{i}^{4 / 3} \hbar \omega$. In this coordinate system $\epsilon=\epsilon_{1}+\epsilon_{2}+\epsilon_{3}$ defines a surface of constant energy $\epsilon$. Then $G(\epsilon)$ is proportional to the volume in the first octant bounded by the surface

$G(\epsilon)=\frac{27}{64(\hbar \omega)^{9 / 4}} \int_{0}^{\epsilon} \epsilon_{1}^{-1 / 4} d \epsilon_{1} \int_{0}^{\epsilon-\epsilon_{1}} \epsilon_{2}^{-1 / 4} d \epsilon_{2} \int_{0}^{\epsilon-\epsilon_{1}-\epsilon_{2}} \epsilon_{3}^{-1 / 4} d \epsilon_{3}$.

To evaluate the integral we use the relation $\int_{0}^{u} x^{\nu-1}(u-x)^{\mu-1}=u^{\mu+\nu-1} B(\mu, \nu)$, where $B(\mu, \nu)=\Gamma(\mu) \Gamma(\nu) / \Gamma(\mu+\nu)$ [13]. Then the density of states

$$
g(\epsilon)=\frac{d G(\epsilon)}{d \epsilon}=0.6852 \frac{\epsilon^{5 / 4}}{(\hbar \omega)^{9 / 4}} .
$$

This expression for density of states is used to calculate $T_{c}$. For bosons, the total number of particles occupying the excited states is

$$
N_{\text {exc }}=\int_{0}^{\infty} \frac{g(\epsilon)}{e^{(\epsilon-\mu) / k T}-1} d \epsilon .
$$

At critical temperature $\mu \rightarrow 0$ in the case of bosons and $N_{\text {exc }}$ is equal to the total number of bosons $N$. Evaluating the integral gives $T_{c}$ in terms of the number of bosons

$$
k T_{c}=\frac{N^{4 / 9} \hbar \omega}{[0.6852 \Gamma(9 / 4) \zeta(9 / 4)]^{4 / 9}} .
$$

The corresponding expression for three dimensional isotropic harmonic oscillator potential is [14]

$$
k T_{c}=\frac{\hbar \omega_{o} N^{1 / 3}}{[\zeta(3)]^{1 / 3}} .
$$

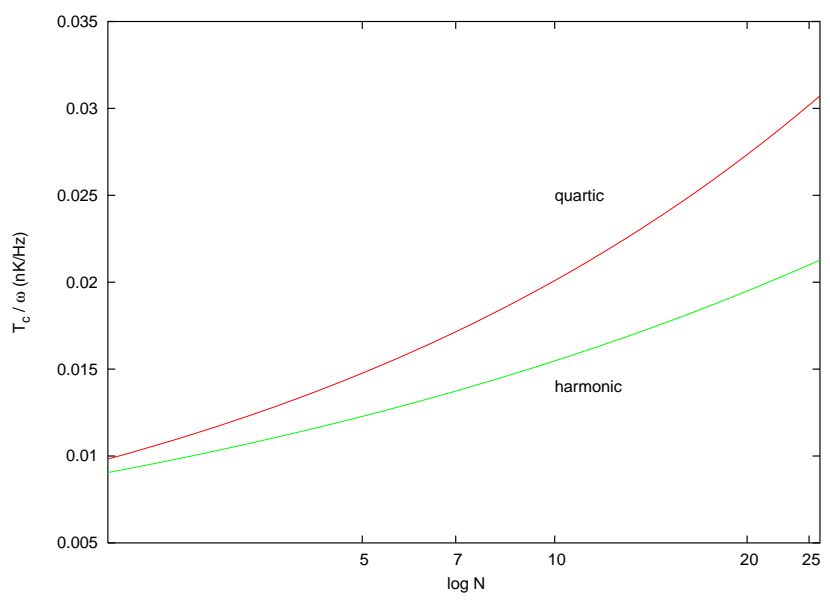

FIG. 1: Ratio of the critical temperature $\left(T_{c}\right)$ to angular frequency $\left(\omega_{o}\right)$ as a function of the number of bosons on the $\log$ scale. Here we have taken $1.389\left(\lambda \hbar^{4} / m^{2}\right)^{1 / 3}=\hbar \omega_{o}\left(\omega_{o}\right.$ is angular frequency of isotropic harmonic oscillator). When $\log N=7$, the $T_{c}$ for the $3 \mathrm{D}$ quartic potential is approximately six times higher than the $3 \mathrm{D}$ isotropic harmonic potential.

From eq. (8) and eq. (9), the ratio of the critical temperatures in the two potentials is

$$
\begin{aligned}
\frac{\left(T_{c}\right)_{\text {quartic }}}{\left(T_{c}\right)_{\text {harmonic }}} & =\frac{N^{4 / 9} \zeta(3)^{1 / 3}}{N^{1 / 3}[0.6852 \Gamma(9 / 4) \zeta(9 / 4)]^{4 / 9}} \\
& =1.006 N^{1 / 9}
\end{aligned}
$$

Where we have taken $\hbar \omega=\hbar \omega_{o}$ to obtain the ratio. The ratio is proportional to $N^{1 / 9}$, which means that for $10^{7}$ atoms, $T_{c}$ in the case of $3 \mathrm{D}$ quartic potential trap is approximately six times higher than that of the $3 \mathrm{D}$ isotropic harmonic trap.

\section{EFFECT OF FINITE PARTICLE NUMBER}

The expression of $T_{c}$ in the previous section is with the approximation that the ground state energy is zero, which is a valid approximation when the system has large number of bosons. For finite number of bosons, the zero point energy causes a change in the value of $T_{c}$. For the $3 \mathrm{D}$ quartic potential, the ground state energy is [12]

$$
\epsilon_{\min }=2.41\left(\frac{\lambda \hbar^{4}}{m^{2}}\right)^{1 / 3}
$$

This should be equal to the change in the chemical potential at the critical temperature, that is $\Delta \mu=\epsilon_{\min }$. As the total number of bosons is fixed [14]

$$
d N=\left(\frac{\partial N}{\partial T}\right)_{\mu} d T+\left(\frac{\partial N}{\partial \mu}\right)_{T} d \mu=0
$$


This implies

$$
\left(\frac{\partial \mu}{\partial T}\right)_{N}=-\left(\frac{\partial N}{\partial T}\right)\left(\frac{\partial N}{\partial \mu}\right)_{T}^{-1}
$$

Using the expression of $N$ at temperatures slightly above $T_{c}$

$$
N=C_{\alpha} \int_{0}^{\infty} \frac{\epsilon^{\alpha-1}}{e^{(\epsilon-\mu) / k T}-1} d \epsilon
$$

This relation is obtained by substituting the general expression for the density of states i.e. $g(\epsilon)=C_{\alpha} \epsilon^{\alpha-1}$ in eq. (17). Here $C_{\alpha}$ is a constant whose value depends on the form of the trapping potential. Then from eq. (13) and eq. (14) we get

$$
\left(\frac{\partial \mu}{\partial T}\right)_{N}=-\alpha \frac{\zeta(\alpha)}{\zeta(\alpha-1)} k
$$

In this expression $\alpha$ should be greater than 2, otherwise the relation is not valid since $\zeta(1)$ diverges. Using this expression, the change in the critical temperature due to the finite particle number is

$$
\Delta T_{c}=-\frac{\zeta(\alpha-1)}{\alpha \zeta(\alpha) k} \Delta \mu
$$

In the case of $3 \mathrm{D}$ quartic potential $\alpha$ is equal to $9 / 4$. Then

$$
\begin{aligned}
\Delta T_{c} & =\frac{-4 \zeta(5 / 4)}{9 \zeta(9 / 4) k} \Delta \epsilon_{\min } \\
& =\frac{-1.071 \zeta(5 / 4)}{\zeta(9 / 4) k}\left(\frac{\lambda \hbar^{4}}{m^{2}}\right)^{1 / 3} .
\end{aligned}
$$

A relative measure of the effect of zero point energy on the critical temperature is the fractional change of the critical temperature. It is the ratio between $\Delta T_{c}$ and $T_{c}$, for the present case

$$
\frac{\Delta T_{c}}{T_{c}}=\frac{-0.9054 \zeta(5 / 4)}{\zeta(9 / 4)}\left(\frac{\lambda \hbar^{4}}{m^{2}}\right)^{1 / 3} \frac{(\Gamma(9 / 4) \zeta(9 / 4))^{4 / 9}}{\hbar \omega N^{4 / 9}} .
$$

Noting that $\left(\lambda \hbar^{4} / m^{2}\right)^{1 / 3}$ is equivalent to $\hbar \omega / 1.389$ we get

$$
\begin{aligned}
\frac{\Delta T_{c}}{T_{c}}= & \frac{-0.6891 \zeta(1.25) N^{-4 / 9}}{\zeta(2.25)^{5 / 9}} \\
& =-2.56 N^{-4 / 9} .
\end{aligned}
$$

For the $3 \mathrm{D}$ isotropic harmonic potential, the fractional change of the critical temperature is

$$
\frac{\Delta T_{c}}{T_{c}}=-0.73 N^{-1 / 3}
$$

If we compare eq. (20) and eq. (21), we find that the percentage decrease in $T_{c}$ is larger in the case of $3 \mathrm{D}$ quartic potential trap for number of particles $\lesssim 80,000$. But the scenario is reversed for number of particles $>80,000$. This is also evident from fig. (2) where cross over point corresponds to the number of particles $\approx 80,000$.

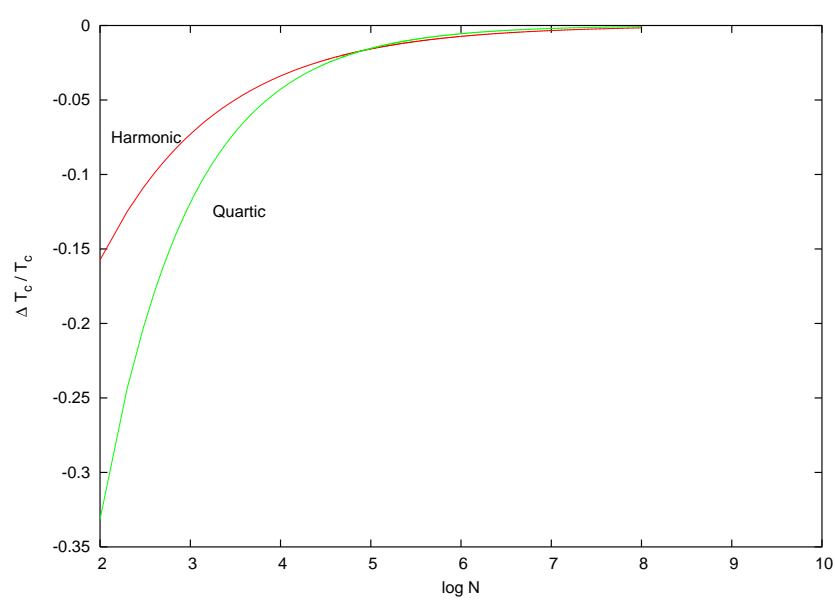

FIG. 2: Fractional change in critical temperature $\left(\Delta T_{c} / T_{c}\right)$ as a function the logarithm of the number of bosons $(\log N$ ). The green and red colored plots correspond to the quartic and quadratic potentials respectively. Below $\log (N)=5$, that is $N \approx 80,000$, the $3 \mathrm{D}$ quartic potential has larger fractional change. Above this point the $3 \mathrm{D}$ isotropic harmonic potential has marginally higher fractional change.

\section{EFFECT OF CROSS TERMS}

\section{A. 3D case}

Consider the general form of the quartic potential, as mentioned earlier

$$
V(\boldsymbol{r})=\lambda(\boldsymbol{r} \cdot \boldsymbol{r})^{2}
$$

In optical traps, it is possible to create confining potentials which are approximately close to this form but a truly spherically symmetric one is not realizable. The difficulty is in producing the cross terms of the potential, for example, terms like $x^{2} y^{2}$ in Cartesian coordinate representation. The absence and presence of the cross terms in quadratic and quartic potentials respectively introduce a key difference between the dynamics in the two potentials. In absence of the cross terms like $x y$, in quadratic potential, a perturbation to the dynamics of a particle along an axis remains confined along that axis. In contrast, it propagates to other axes in the case of quartic potential. For condensates in traps, an important parameter which reflects the effects of these terms is the critical temperature.

Semiclassically, total number of states available to the system can be obtained by dividing the total phase space volume by $h^{3}$, the volume of a single state

$$
\begin{aligned}
G(\epsilon) & =\frac{1}{h^{3}} \int d \mathbf{x} \int d \mathbf{p}, \\
& =\frac{16 \pi^{2}}{h^{3}} \int_{0}^{r^{*}} r^{2} d r \int_{0}^{p^{*}} p^{2} d p .
\end{aligned}
$$

In the above equation $r^{*}$ and $p^{*}$ are radial coordinate and momentum corresponding to the classical turning 
point respectively. Transforming the variable of integration from $p$ to $\epsilon$ (using the relation $p^{2} / 2 m=\epsilon-V(\boldsymbol{r})$ ) we get

$$
G(\epsilon)=\frac{16 \pi^{2} m}{h^{3}} \int_{0}^{r^{*}} r^{2} d r \int_{0}^{\epsilon^{*}} \sqrt{2 m(\epsilon-V(\boldsymbol{r}))} d \epsilon .
$$

Thus the density of states is

$$
g(\epsilon)=\frac{16 \pi^{2} m}{h^{3}} \int_{0}^{r^{*}} r^{2} \sqrt{2 m\left(\epsilon-\lambda r^{4}\right)} d r .
$$

Substituting $r^{4}=x$ we can transform the integral into a form which can be evaluated analytically 13 .

$$
\begin{aligned}
g(\epsilon) & =\frac{4 \pi^{2} m^{3 / 2} \sqrt{2 \lambda}}{h^{3}} \int_{0}^{\epsilon / \lambda}\left(\sqrt{\frac{\epsilon}{\lambda}-x}\right) x^{-1 / 4} d x \\
& =\frac{4 \sqrt{2} \pi^{2} m^{3 / 2} \Gamma(3 / 2) \Gamma(3 / 4) \epsilon^{5 / 4}}{h^{3} \lambda^{3 / 4} \Gamma(9 / 4)}
\end{aligned}
$$

Using this expression for the density of states in eq. (7) we get

$$
k T_{c}=\frac{N^{4 / 9}}{[\Gamma(9 / 4) \zeta(9 / 4)]^{4 / 9}}\left[\frac{h^{3} \lambda^{3 / 4} \Gamma(9 / 4)}{\Gamma(3 / 2) \Gamma(3 / 4) 4 \sqrt{2} \pi^{2} m^{3 / 2}}\right]^{4 / 9}
$$

Comparing with eq. (8) we can obtain the ratio of $T_{c}$ in the two cases, with and without the cross terms, for the $3 \mathrm{D}$ isotropic quartic potential. It is found that $T_{c}$ with the cross terms is 1.2 times higher. This rise in $T_{c}$ can be attributed to the contribution from the cross terms which were neglected while deriving eq. (8). The reason for the difference is, when $g(\epsilon)=C_{\alpha} \epsilon^{\alpha-1}$ then $T_{c}$ varies as $1 / C_{\alpha}{ }^{1 / \alpha}$. Hence, the lower $T_{c}$ in $3 \mathrm{D}$ quartic potentials without the cross terms is due to the higher value of $C_{\alpha}$.

\section{B. 2D case}

In the $2 \mathrm{D}$ case, neglecting the cross terms, the potential is of the form

$$
V(x, y)=\lambda\left(x^{4}+y^{4}\right)
$$

Using the same approach as adopted in the 3D case, the total number of states available to the system is

$$
\begin{aligned}
G(\epsilon) & =\frac{9}{16(\hbar \omega)^{3 / 2}} \int_{0}^{\epsilon} \epsilon_{1}^{-1 / 4} d \epsilon_{1} \int_{0}^{\epsilon-\epsilon_{1}} \epsilon_{2}^{-1 / 4} d \epsilon_{2} \\
& =\frac{3 \Gamma(3 / 4) \Gamma(7 / 4)}{4(\hbar \omega)^{3 / 2} \Gamma(5 / 2)} \epsilon^{3 / 2}
\end{aligned}
$$

Thus the density of states

$$
g(\epsilon)=\frac{0.9531 \epsilon^{1 / 2}}{(\hbar \omega)^{3 / 2}} .
$$

Substituting this expression of $g(\epsilon)$ in eq. (7) we get

$$
k T_{c}=\frac{\hbar \omega N^{2 / 3}}{[\zeta(3 / 2) \Gamma(3 / 2) 0.9531]^{2 / 3}} .
$$

The corresponding expression when cross terms are considered is [1]

$$
k T_{c}=\left[\frac{N h^{2} \sqrt{\lambda}}{2 \pi^{2} m \Gamma(3 / 2) \zeta(3 / 2)}\right]^{2 / 3} .
$$

Comparing eq. (34) and eq. (35) we find that $T_{c}$ with the cross terms in eq. (35) is approx. 1.12 times higher than $T_{c}$ without the cross terms in eq. (34). Thus the cross terms increase $T_{c}$ in $2 \mathrm{D}$ as well as $3 \mathrm{D}$ case.

\section{CONCLUSIONS}

Our calculations show that $T_{c}$ in the case of the $3 \mathrm{D}$ quartic potential trap is higher than that of the isotropic harmonic potential trap. This is due to the form of the density of states $g(\epsilon)$, which varies as $\epsilon^{5 / 4}$ and $\epsilon^{2}$ in 3D isotropic quartic and quadratic trapping potentials respectively. This implies lower density of states in quartic oscillator potential compared to isotropic harmonic oscillator potential. However, more interesting is the effect of the cross terms. In the 3D isotropic harmonic potential trap the cross terms are absent, which is not the case for the $3 \mathrm{D}$ quartic potential trap. The cross terms tend to decrease the density of states and raise $T_{c}$. These terms increase $T_{c}$ by factor of 1.2 and 1.1 in the $3 \mathrm{D}$ and $2 \mathrm{D}$ quartic trap potentials respectively as compared to the case without the cross terms. Experimentally, in optical traps, the potentials without the cross terms are more appropriate. We find that the effect of finite particle number is more pronounced in the $3 \mathrm{D}$ quartic potential when the number of particles is $<10^{5}$. The situation is reversed when the number of particles is $\gtrsim 10^{5}$. The cause of the reversal lies in the form of the fractional change $\Delta T_{c} / T_{c}$ for the two potentials. The ratio of the fractional change between $3 \mathrm{D}$ isotropic quartic potential to harmonic potential is $3.51 / N^{1 / 9}$. It is $\approx 1$ for $N \approx 10^{5},>1$ for $N<10^{5}$ and $<1$ for $N \gtrsim 10^{5}$. Thus, when $N<10^{5}$ the constant factor is dominant in eq. (20) and is responsible for the larger value of $\Delta T_{c} / T_{c}$ in quartic potential. But when $N \gtrsim 10^{5}, N^{-4 / 9}$ dominates and $\Delta T_{c} / T_{c}$ of the quartic potential is lower than that of the harmonic potential. 
[1] M. Greiner, O. Mandel, T. Esslinger, T. W. Hänsch and I. Bloch, Nature 415, 39 (2002).

[2] O. Gygi, H. G. Katzgraber, M. Troyer, S. Wessel and G. G. Batrouni, Phys. Rev. A 73, 063606 (2006).

[3] A. L. Fetter, Phys. Rev. A. 64, 063608 (2001).

[4] T. K. Ghosh, Phys. Rev. A. 69, 043606 (2004).

[5] J. Kim and A. L. Fetter, Phys. Rev. A. 72, 023619 (2005).

[6] I. Danaila, Phys. Rev. A. 72, 013605 (2005).

[7] A. L. Fetter, B. Jackson and S. Stringari, Phys. Rev. A. 71, 013605 (2005).

[8] S. Bargi, G. M. Kavoulakis and S. M. Reimann, Phys. Rev. A. 73, 033613 (2006).
[9] S. Kling and A. Pelster, Phys. Rev. A 76, 023609 (2007).

[10] V. Bretin, S. Stock, Y. Seurin and J. Dalibard, Phys. Rev. Lett. 92, 050403 (2004).

[11] V. Bagnato and D. Kleppner, Phys. Rev. A. 44, 7439 (1991).

[12] P. M. Mathews, M. Seetharaman and S. Raghavan, J. Phys. A 15, 103 (1981).

[13] I. S. Gradshteyn and I. M. Ryzhik, Table of Integral Series And Products. Academic Press, sixth edition, 2001.

[14] C. J. Pethick and H. Smith, Bose-Einstein Condensation in Dilute Gases Cambridge University Press, 2002. 